\title{
Método Mehlich 3 como substituinte ao HCl para extração de Cobre e Zinco em solos com alto teor de matéria orgânica do sul do Brasil
}

\author{
Mehlich 3 method as substituent to the HCl method for extraction of Copper and Zinc in soils from \\ Southern Brazil with high levels of organic matter
}

\author{
Evandro Luiz Schoninger ${ }^{\mathrm{I}}$ Luciano Colpo Gatiboni ${ }^{\mathrm{II}}$ Daniel Linhares ${ }^{\mathrm{III}}$
}

\begin{abstract}
- NOTA -
RESUMO

No presente trabalho, foram comparados dois métodos de extração de cobre (Cu) e zinco ( $\mathrm{Zn})$ em solos com teor de matéria orgânica (MO) maior que $50 \mathrm{~g} \mathrm{~kg}^{-1}$. Os métodos de extração comparados foram: Extração com $\mathrm{HCl} 0,1 \mathrm{~mol} \mathrm{~L}^{-1}$ (método padrão para os estados do Rio Grande do Sul e Santa Catarina) e Mehlich 3. Este trabalho foi realizado no Laboratório de Química e Fertilidade do Solo da Universidade do Estado de Santa Catarina (UDESC), no ano de 2009. As

(UDESC), in 2009. The two extraction methods were applied to 286 soil samples from the Soil Analysis Public Laboratory of UDESC, all with more than $50 \mathrm{~g} \mathrm{~kg}^{-1} \mathrm{OM}$. The copper and zinc in extracts were determined by flame atomic absorption spectrometry. The results showed significant correlation between the methods, $\mathrm{Cu}(r=0.80)$ and $\mathrm{Zn}(r=0.93)$. The solution Mehlich 3 extracted more copper and less zinc when compared to the solution of $\mathrm{HCl} 0.1 \mathrm{~mol} \mathrm{~L}^{-1}$. The Mehlich 3 proved to be efficient in the extraction of copper and zinc in soils with high OM content, which may replace the current method.
\end{abstract} duas metodologias de extração foram aplicadas em 286 amostras de solo provenientes do Laboratório de Análise do Solo da UDESC, todas com mais de $50 \mathrm{~g} \mathrm{~kg}^{-1}$ de MO. Os teores de cobre e zinco nos extratos foram determinados por espectrometria de absorção atômica com chama. Os resultados mostraram que houve correlação significativa entre os métodos, $\mathrm{Cu}(r=0,80)$ e $\mathrm{Zn}(r=0,93)$. A solução de Mehlich 3 extraiu mais cobre e menos zinco, quando comparada à solução de $\mathrm{HCl} 0,1 \mathrm{~mol} \mathrm{~L}^{-1}$. O Mehlich 3 demonstrou ser eficiente na extração de cobre e zinco em solos com alto teor de $\mathrm{MO}$, podendo substituir o método atual.

Palavras-chave: análise de solo, micronutrientes, extratores.

\section{ABSTRACT}

In this study were compared two methods of extraction of copper $(\mathrm{Cu})$ and zinc $(\mathrm{Zn})$ in soils with organic matter (OM) higher than $50 \mathrm{~g} \mathrm{~kg}^{-1}$. The extraction methods compared were: Extraction with $\mathrm{HCl} 0.1 \mathrm{~mol} \mathrm{~L}^{-1}$ (standard method for the states of Rio Grande do Sul and Santa Catarina) and Mehlich 3. This work was performed at the Laboratory of Soil Chemistry and Fertility, Santa Catarina State University
Key words: soil analysis, micronutrients, extractants.

As práticas de manejo da fertilidade a serem adotadas em áreas agrícolas dependem, dentre muitos fatores, da atual capacidade que o solo apresenta em fornecer nutrientes às plantas. Aproximações desta capacidade são obtidas através da análise química de amostras de solo por meio de métodos mais adequados e calibrados para cada região. Entretanto, para que um método seja adotado como padrão em laboratórios de análise de solo, ele deve apresentar boa correlação com os teores de nutrientes absorvidos pelas plantas (BATAGLIA \& RAIJ, 1989), além de ser eficiente, não poluente e de baixo custo. Nesse sentido, métodos que extraem simultaneamente vários elementos químicos de interesse podem trazer o benefício da redução dos custos e do tempo para análise.

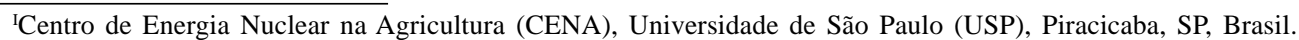

"Programa de Pós-graduação em Manejo do Solo, Universidade do Estado de Santa Catarina (UDESC), 88520-000, Lages, SC, Brasil. E-mail: lgatiboni@gmail.com. Autor para correspondência.

IIICurso de Agronomia, UDESC, Lages, SC, Brasil.
} 
Como há a busca por soluções extratoras multielementares que apresentem boa correlação com os teores extraídos pelos métodos atuais, e que atendam à maioria dos requisitos necessários para um bom extrator, o meio científico tem realizado várias pesquisas com o método multielementar Mehlich 3 (BRENANN et al., 2008; BORTOLON \& GIANELLO, 2009; BORTOLON \& GIANELLO, 2010). O método Mehlich 3 (MEHLICH, 1984) tem sido amplamente estudado por extrair simultaneamente $\mathrm{P}, \mathrm{K}, \mathrm{Ca}, \mathrm{Mg}$, $\mathrm{Mn}, \mathrm{Fe}, \mathrm{Cu}$ e $\mathrm{Zn}$, tornado-o versátil para uso em laboratórios de rotina de análise de solo.

Para o caso de Cu e Zn, BORTOLON \& GIANELLO (2009) avaliaram vários métodos de extração desses nutrientes e encontraram elevado coeficiente de correlação para os teores de $\mathrm{Cu}(0,94$ e 0,89) e Zn $(0,84$ e 0,94) extraídos por Mehlich 3 e o HCl, que é método padrão utilizado nos estados do Rio Grande do Sul e Santa Catarina. De modo semelhante, CONSOLINI \& COUTINHO (2004) verificaram que o Mehlich 3 foi tão eficiente quanto o $\mathrm{HCl}$ para extração de $\mathrm{Zn}$, mesmo em solos com variação no pH. Contudo, os elementos $\mathrm{Cu}$ e $\mathrm{Zn}$ merecem atenção especial em solos com elevado teor de matéria orgânica, pois apresentam afinidade de adsorção à fração orgânica, principalmente o $\mathrm{Cu}$ (ABREU et al., 2007), tornando-se necessária a avaliação da efetividade da solução Mehlich 3 na extração destes nutrientes nesta condição. O objetivo deste trabalho foi avaliar a capacidade de extração de $\mathrm{Cu}$ e $\mathrm{Zn}$ pela solução de Mehlich 3 em solos com teor de matéria orgânica (MO) maior que $50 \mathrm{~g} \mathrm{~kg}^{-1}$.

O trabalho foi realizado no Laboratório de Química e Fertilidade do Solo do CAV - UDESC, no ano de 2009, com 286 amostras de solos dos estados de Santa Catarina e Rio Grande do Sul, provenientes do banco de amostras de solo do Laboratório de Análise do Solo (CAV - UDESC), selecionadas quanto ao teor de matéria orgânica $\left(>50 \mathrm{~g} \mathrm{~kg}^{-1}\right)$. Os valores médios de $\mathrm{pH}$ do solo, bem como os teores médios de argila e matéria orgânica das amostras, determinados pelas metodologias descritas em TEDESCO et al. (1995) são apresentados na tabela 1 .

As amostras de solo foram submetidas à

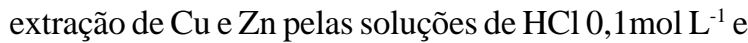
de Mehlich 3. Na extração com $\mathrm{HCl}$, descrita por TEDESCO et al. (1995), foi utilizada a relação solo:solução de 1:4, com agitação horizontal a 120 rotações por minuto durante 30 minutos e permanência em repouso durante as 16 horas seguintes para decantação da fase sólida, com posterior retirada de uma alíquota do extrato sobrenadante. Para a extração com Mehlich 3, foi utilizada a relação solo:solução de 1:10, com agitação horizontal a 120 rotações por minuto durante 5 minutos. A filtração do extrato descrita por MEHLICH (1984) foi substituída pelo processo de decantação por 16 horas (BORTOLON \& GIANELLO, 2010), para posterior coleta de uma alíquota do extrato sobrenadante. Os teores de $\mathrm{Cu}$ e $\mathrm{Zn}$ nos extratos foram determinados por espectrometria de absorção atômica. Os teores de cobre e zinco obtidos por $\mathrm{HCl}$ e Mehlich 3 foram submetidos ao teste de correlação de Pearson e também foram estabelecidas regressões lineares entre os teores extraídos pelas soluções.

Os teores máximos, mínimos, médios e a mediana de Cu e Zn extraídos pelos dois métodos são apresentados na tabela 1. Ambos os extratores proporcionaram grande variação entre os valores máximos e mínimos para cada nutriente. Essa variação, provavelmente, está relacionada com particularidades de cada solo avaliado, devido ao elevado número de amostras provenientes de uma região com presença de classes de solos distintas.

Houve correlação significativa entre os teores de $\mathrm{Cu}$ extraídos pelas soluções de $\mathrm{HCl}$ e Mehlich 3 (Figura 1a). Para este nutriente, o coeficiente

Tabela 1 - Valores médios e amplitude do pH, dos teores de argila e matéria orgânica (MO) dos solos analisados e dos teores de Cu e Zn extraídos por $\mathrm{HCl}$ e Mehlich 3.

\begin{tabular}{|c|c|c|c|c|c|c|c|}
\hline Parâmetros & $\mathrm{pH}^{1}$ & Argila & MO & $\mathrm{Cu}-\mathrm{HCl}^{2}$ & $\mathrm{Cu}-\mathrm{M}^{3}{ }^{3}$ & $\mathrm{Zn}-\mathrm{HCl}^{2}$ & $\mathrm{Zn}-\mathrm{M3}^{3}$ \\
\hline & $(1: 1)$ & \multicolumn{2}{|c|}{------------------g dm³ ---------------- } & \multicolumn{4}{|c|}{ 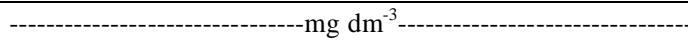 } \\
\hline Mínimo & 3,1 & 80 & 50 & 0,0 & 0,0 & 0,0 & 0,0 \\
\hline Máximo & 7,4 & 650 & 120 & 20,0 & 27,5 & 70,4 & 53,0 \\
\hline Média & 5,4 & 330 & 59 & 3,8 & 5,7 & 4,8 & 3,4 \\
\hline Mediana & 5,4 & 320 & 55 & 3,2 & 5,4 & 2,5 & 1,7 \\
\hline
\end{tabular}

${ }^{1}$ - pH em água (TEDESCO et al., 1995).

- $\mathrm{HCl}=$ Solução de $\mathrm{HCl}$ 0,1 mol L-1 (TEDESCO et al., 1995).

- M3 = Solução de Mehlich 3 (MEHLICH, 1984). 
de correlação foi de 0,80 , no qual o Mehlich 3 extraiu, de maneira geral, $1,94 \mathrm{mg} \mathrm{dm}^{-3}$ a mais que o $\mathrm{HCl}$. Segundo SIMS (1989) e BORTOLON \& GIANELLO (2010), o método Mehlich 3 extrai mais cobre devido à formação de complexos estáveis (quelatos) entre o cobre e o EDTA empregado nesta solução. Este mecanismo é a provável explicação para o fenômeno observado na figura 1a, em que alguns solos apresentaram teores de Cu de aproximadamente zero pelo extrator $\mathrm{HCl}$ e próximo de $5 \mathrm{mg} \mathrm{dm}{ }^{-3}$ pelo extrator Mehlich 3. Este poder de extrair mais cobre poderá facilitar a determinação deste nutriente em solos com teores extremamente baixos, além de indicar uma possível substituição do método atual pelo Mehlich 3 com ajustes pela equação, já que o coeficiente angular da equação é 1,0 , facilitando a interpretação dos teores extraídos pelo método alternativo. De acordo com essa equação, as classes de interpretação dos níveis de $\mathrm{Cu}$ no solo, com arredondamento, passariam de $<0,2,0,2-0,4$, e $>0,4 \mathrm{mg}$ $\mathrm{dm}^{-3}$ (CQFS RS/SC, 2004) para <2,2, 2,2 - 2,4, > 2,4mg $\mathrm{dm}^{-3}$ para os níveis baixo, médio e alto, respectivamente.

Os teores de $\mathrm{Zn}$ extraídos pelos dois métodos apresentaram correlação significativa (Figura $1 b$ e 1c). No entanto, foi observada grande concentração de pontos abaixo do teor de $20 \mathrm{mg} \mathrm{dm}^{-3}$ de $\mathrm{Zn}$ (Figura 1b). Por isso, na figura 1c, optou-se pela exclusão dessas amostras com teores extraídos por $\mathrm{HCl}$ acima de $20 \mathrm{mg} \mathrm{dm}^{-3}$, já que há menor possibilidade de se encontrar esses teores em solos agricultáveis, e a pequena porcentagem das amostras totais que estes pontos representam $(<3,5 \%)$ no presente trabalho. Desse modo, o coeficiente de correlação de extração de Zn entre os métodos foi de 0,93 ( $\mathrm{P}<0,01)$, em que, de maneira geral, o Mehlich 3 extraiu aproximadamente 66 \% da quantidade de Zn extraída por HCl, como também observado por CONSOLINI \& COUTINHO (2004). Corroborando esses resultados, BORTOLON \& GIANELLO (2010) encontraram teores de zinco extraído

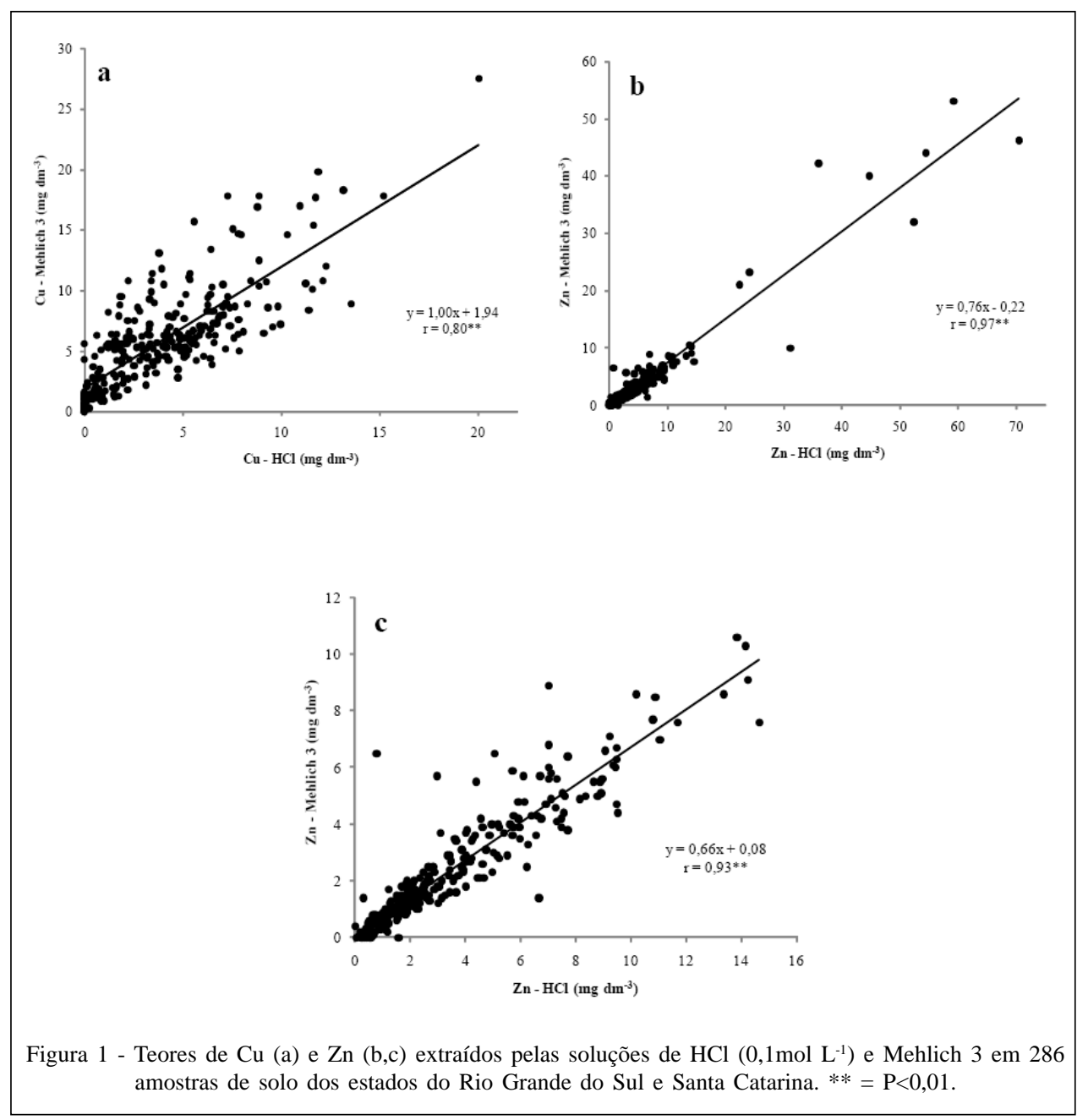

Ciência Rural, v.42, n.7, jul, 2012. 
por Mehlich 3, em média, 50\% menores que os extraídos por HCl em 130 amostras de solo do Estado do Rio Grande do Sul. Enquanto o $\mathrm{Cu}$ no solo tem alta afinidade pela matéria orgânica, como discutido anteriormente, o zinco encontra-se no solo adsorvido à matéria orgânica e também aos carbonatos e óxidos de ferro e alumínio (BORTOLON \& GIANELLO, 2009). Com isso, provavelmente, a maior acidez da solução de $\mathrm{HCl}(\mathrm{pH}$ 1,0) que a do Mehlich 3 (pH 2,5) provoca maior dissolução do Zn dos coloides inorgânicos, quantificando mais Zn que a solução de Mehlich 3, como constatado por CONSOLINI \& COUTINHO(2004).

De modo contrário ao ocorrido para o $\mathrm{Cu}$, essa menor extração poderá dificultar a determinação de Zn em solos com teores muito baixos. Aplicando-se a equação da figura 1c sobre os valores-limite das classes de disponibilidade de $\mathrm{Zn}$ pelo extrator $\mathrm{HCl}$ usadas atualmente (CQFS RS/SC, 2004), pode-se propor novas classes de interpretação da disponibilidade de Zn no solo quando utilizado o Mehlich 3, alterando-as de $<0,2,0,2-0,5$, e $>0,5 \mathrm{mg} \mathrm{dm}^{-3}$, para $<0,2,0,2-0,4$, e $>0,4 \mathrm{mg} \mathrm{dm}^{-3}$, para os níveis baixo, médio e alto, respectivamente.

Os teores de Cu e Zn extraídos pela solução extratora de Mehlich 3 apresentam correlação com os extraídos pela solução de $\mathrm{HCl}$ e, por isso, conclui-se que o Mehlich 3 é eficiente na extração dos elementos avaliados em solos com elevado teor de matéria orgânica.

\section{REFERÊNCIAS}

ABREU, C.A. et al. Micronutrientes. In: NOVAIS, R.F. et al. Fertilidade do Solo. Viçosa: Sociedade Brasileira de Ciência do Solo, 2007. Cap.11, p.645-736.

BATAGLIA, O.C.; RAIJ, B. van. Eficiência de extratores de micronutrientes na análise de solo. Revista Brasileira de Ciência do Solo, v.13, p.205-212, 1989.

BORTOLON, L.; GIANELLO, C. Disponibilidade de cobre e zinco em solos do sul do Brasil. Revista Brasileira de Ciência do Solo, v.33, p.647-658, 2009. Disponível em: <http:// www.scielo.br/scielo.php?script=sci_issuetoc $\&$ pid $=0100$ 068320090003\&lng=pt\&nrm =iso>. Acesso em: 08 mar. 2012. doi: 10.1590/S0100-06832009000300017.

BORTOLON, L.; GIANELLO, C. Extração de cobre e de zinco por soluções multielementares em solos do sul do Brasil. Ciência Rural, v.40, p.670-673, 2010. Disponível em: <http:/ /www.scielo.br/scielo.php?script=sci_arttext\&pid=S010384782010000300029>. Acesso em: 08 mar. 2012. doi: $10.1590 / \mathrm{S} 0103-84782010005000035$.

BRENANN, D. et al. Evaluation of Mehlich 3 for Extraction of Copper and Zinc from Irish Grassland Soils and for Prediction of Herbage Content. Communications in Soil Science and Plant Analysis, v.39, p.1943-1962, 2008. Disponível em: <http://www.tandfonline.com/doi/abs/10.1080/ 00103620802134743>. Acesso em: 08 mar. 2012. doi: 10.1080/00103620802134743.

COMISSÃO DE QUÍMICA E FERTILIDADE DO SOLO CQFS RS/SC. Manual de adubação e calagem para os estados do Rio Grande do Sul e Santa Catarina. Porto Alegre: SBCS/NRS/UFRGS, 2004. 400p.

CONSOLINI, F.; COUTINHO, E.L.M. Efeito da aplicação de $\mathrm{Zn}$ e do $\mathrm{pH}$ do solo na disponibilidade do micronutriente. Acta Scientiarum Agronomy, v.26, p.7-12, 2004. Disponível em: <http://periodicos.uem.br/ojs/index.php/ActaSciAgron/ article/view/1948>. Acesso em: 08 mar. 2012. doi: 10.4025/ actasciagron.v26i1.1948.

MEHLICH, A. Mehlich 3 soil test extractant: a modification of Mehlich 2 extractant. Communications in Soil Science and Plant Analysis, v.15, p.1409-1416, 1984. Disponível em: <http://www.tandfonline.com/doi/abs/10.1080/ 00103628409367568>. Acesso em: 08 mar. 2012. doi: 10.1080/00103628409367568.

SIMS, J.T. Comparison of Mehlich 1 and Mehlich 3 extractants for P, K, Ca, Mg, Mn, Cu and $\mathrm{Zn}$ in Atlantic coastal plain soils. Communications in Soil Science and Plant Analysis, v.20, p.1707-1726, 1989. Disponível em: <http:// www.tandfonline.com/doi/abs/10.1080/00103628909368178>. Acesso em: 08 mar. 2012. doi: 10.1080/00103628909368178.

TEDESCO, M.J. et al. Análise de solo, plantas e outros materiais. Porto Alegre: Universidade Federal do Rio Grande do Sul, Departamento de Solos, 1995. 147p. (Boletim Técnico, 5). 